\title{
Discours
}

Revue de linguistique, psycholinguistique et

informatique. A journal of linguistics, psycholinguistics and computational linguistics

$23 \mid 2018$

Varia

\section{La littératie en voie de changement : respect du genre et jonctions interpropositionnelles dans des lettres à des professionnels du droit sur Internet}

\section{Soili Hakulinen et Meri Larjavaara}

\section{OpenEdition \\ Journals}

Édition électronique

URL : http://journals.openedition.org/discours/9818

DOI : $10.4000 /$ discours. 9818

ISSN : 1963-1723

Éditeur :

Laboratoire LATTICE, Presses universitaires de Caen

\section{Référence électronique}

Soili Hakulinen et Meri Larjavaara, « La littératie en voie de changement : respect du genre et jonctions interpropositionnelles dans des lettres à des professionnels du droit sur Internet », Discours [En ligne], 23 | 2018, mis en ligne le 21 décembre 2018, consulté le 13 juin 2019. URL : http://

journals.openedition.org/discours/9818; DOI : 10.4000/discours.9818 

Revue de linguistique, psycholinguistique et informatique

\title{
La littératie en voie de changement : respect du genre et jonctions interpropositionnelles dans des lettres à des professionnels du droit sur Internet
}

\author{
Soili Hakulinen \\ Université de Tampere \\ Meri Larjavaara \\ Université d'Åbo Akademi
}

Soili Hakulinen, Meri Larjavaara, «La littératie en voie de changement: respect du genre et jonctions interpropositionnelles dans des lettres à des professionnels du droit sur Internet», Discours [En ligne], 23 | 2018, mis en ligne le 21 décembre 2018. 



\title{
La littératie en voie de changement : respect du genre et jonctions interpropositionnelles dans des lettres à des professionnels du droit sur Internet
}

\author{
Soili Hakulinen \\ Université de Tampere \\ Meri Larjavaara \\ Université d'Åbo Akademi
}

\begin{abstract}
Les conventions de l'écriture du français font l'objet de cet article. La démocratisation de l'écriture - tout locuteur écrit et tout locuteur peut rendre ses textes publics sur Internet - est supposée être le catalyseur de changements des normes du français écrit. Pour travailler sur la question, nous étudions un échantillon de lettres provenant d'un site Internet d'aide juridique, écrites par des internautes et destinées à des professionnels du droit. Les lettres étudiées présentent une cohérence claire qui suit un certain modèle. Nous constatons la présence de phénomènes appartenant au français écrit conventionnel mais aussi celle de caractéristiques qui s'en éloignent. Nous nous sommes particulièrement concentrées sur l'étude des jonctions entre propositions qui ont un rôle cohésif. En utilisant les notions proposées par Raible (1992; 2001), nous avons étudié le degré d'intégration du texte et sa densité. Il apparaît que le genre discursif en question est à la charnière de tendances presque opposées: la densité du français écrit conventionnel est confrontée à de multiples moyens linguistiques plus lâches, témoins d'un desserrement textuel.

Mots clés: français, normes, langue écrite, discours numérique, tradition discursive, genre, jonction

The article deals with conventions of written French. Democratization of writing - every speaker writes and everyone can publish texts on the Internet - is seen as a catalyst for the change of norms of written French. To investigate this phenomenon we analyse letters published on an online legal advice forum. The letters are written by Internet users to professional lawyers. They are clearly coherent and follow a certain structure. Some of their characteristics pertain to standard written French while others do not. In our study, we concentrate particularly on junctions between propositions. These junctions have an important role in cohesion. By taking advantage of notions introduced by Raible (1992; 2001), we study the integrative features of the text and its density. It seems that the discursive genre in question lies at the crossroads of two almost opposite tendencies: the density found in standard written French is confronted with less dense linguistic features.
\end{abstract}

Keywords: French, norms, written language, digital discourse, discourse tradition, genre, junction

\section{Introduction ${ }^{1}$}

Nous assistons actuellement à une «démocratisation» de l'écriture: dans nos sociétés modernes, nous écrivons plus que jamais et, en même temps, une partie de plus

1. Nous remercions Luciane Hakulinen ainsi que nos deux relecteurs anonymes pour leurs remarques pertinentes. 
en plus importante des textes que nous lisons ne sont plus des textes imprimés. En plus des SMS, chats et messages WhatsApp à usage privé qui font partie de la communication quotidienne de pratiquement tout le monde de nos jours, n'importe qui peut faire diffuser ses écrits par voie numérique - Internet avec ses réseaux sociaux - auprès du monde entier, virtuellement, sans que quiconque ne les révise ou ne les critique au préalable. Ce type d'écriture est d'ailleurs souvent considéré comme une forme intermédiaire entre la communication orale et l'écriture telle qu'elle a été vue au cours des siècles (voir Hakulinen, 2003; Herring et al., 2013; Marcoccia, 2016: 70-75). Nous pouvons nous demander à juste titre si ces écrits «de tous les jours», diffusés par ordinateur, imprégnés d'oralité, ne sont pas en train de transformer le panorama de la culture écrite dans nos sociétés.

Il est bien connu que la norme écrite jouit d'une estime inégalée dans la société française et que le grand public a adopté une position fortement prescriptive envers la langue; la discussion autour des réformes de l'orthographe au printemps 2016 en témoigne. La langue française est considérée comme un symbole de l'identité nationale ${ }^{2}$. Cette attitude quelque peu exceptionnelle parmi les autres pays européens est attribuée principalement à trois facteurs (Lodge, 1997: 307-309), qui contribuent chacun à leur façon au renforcement du prestige des normes de la langue écrite en France: le système scolaire, le riche patrimoine littéraire ainsi que l'engagement de l'État et de ses organismes officiels pour maintenir les normes traditionnelles.

Si, en 1993, Lodge (1997: 309) considère que les mots de Brunot dans les années 1920 - «le règne de la grammaire [...] a été, en France, plus tyrannique et plus long qu'en aucun autre pays» (Brunot, 1966: 4) - n'ont rien perdu de leur actualité, nous pouvons, à la fin des années 2010, nous demander si ce fameux règne n'est pas finalement en train de s'effriter. Serions-nous en train de passer d'une société où la culture «de prestige» doit se manifester à travers des textes écrits, soignés, revus, corrigés, édités et publiés, à une culture écrite qui laisse davantage place à des écrits spontanés, plus ou moins influencés par la langue orale ${ }^{3}$ ? La norme semble se relâcher, l'écriture n'étant plus uniquement l'apanage des personnes cultivées ayant appris à maîtriser la norme littéraire lors d'études poussées. Même la littérature témoigne de la prolifération de normes possibles, et on peut parler de «l'émancipation littéraire de la norme grammaticale» (Philippe et Piat, 2009: 48 et passim).

L'intérêt du grand public pour la préservation de la «bonne langue» n'a cependant pas diminué, ce qui peut être constaté par le nombre des discussions et débats publics

2. Paveau et Rosier (2008: 261) le formulent ainsi : «Dans l’imaginaire linguistique français [...], le génie de la langue française correspond à des caractéristiques attribuables à la France et à ses habitants: c'est le lien si souvent fait entre la langue d'un peuple et son identité culturelle, entre le caractère langagier et le caractère national».

3. Ferguson (1972) a introduit en sociolinguistique la notion de «diglossie» : une société peut connaitre (au moins) deux variétés de langue, l'une «haute», standardisée, qui a produit une littérature, et qui jouit d'un prestige dans la société. Cette variété s'acquiert à travers une éducation formelle. L'autre variété est «basse», et elle s'emploie dans les situations privées et peu formelles. 
sur le sujet ${ }^{4}$. La situation est quelque peu paradoxale : le public français veut que la norme écrite traditionnelle soit préservée, mais les locuteurs capables de la maittriser parfaitement sont rares ou, du moins, l'apprentissage de cette norme demande des efforts considérables aux enfants, ce que montre aussi la comparaison de l'école en France avec d'autres pays européens ${ }^{5}$; la capacité d'écrire selon les conventions continue à être un facteur socialement discriminant en France (voir Walsh, 2016: 233). Une écriture plus spontanée et informelle semble pourtant se généraliser, ce qui entraînera certainement un changement dans les conventions acceptables; de ce fait, les personnes capables d'écrire selon une norme acceptable deviendront de plus en plus nombreuses et l'écriture ne sera plus aussi exclusive.

Dans cet article, la question du changement des conventions de l'écriture dans la société française sera étudiée à travers un échantillon de lettres provenant d'un site Internet d'aide juridique, écrites par des internautes et destinées à des professionnels du droit. Nous sommes parties du principe que le domaine du droit implique une surveillance accrue des scripteurs sur leurs écrits, ce qui constitue un bon observatoire de la norme et des conventions acceptables. Le point de vue sera la réalisation du genre attendu dans les lettres ainsi que la nature des jonctions interpropositionnelles, qui sera considérée comme caractéristique de ce discours.

Dans la section suivante seront introduites des notions centrales de l'article: celle de genre discursif et sa relation avec celle de tradition discursive, ainsi que celle de densité textuelle. La section 3 présentera le corpus. La section 4 commencera par discuter, en 4.1, la cohérence des lettres: on y constatera que le genre y est respecté. La cohésion fera l'objet de la section 4.2, où les jonctions seront analysées. La section 5 reviendra sur la densité des textes et l'article se terminera par une conclusion (section 6).

\section{2. À la recherche du genre}

Les lettres étudiées appartiennent à un certain genre discursif, celui des lettres envoyées à un forum sur Internet qui a comme objectif de relier le public et les professionnels. Les genres sont vus ici comme des pratiques (socio-)discursives

4. La polémique en 2016 au sujet de l'application des rectifications de l'orthographe de 1990 en est un exemple (http://www.lemonde.fr/les-decodeurs/article/2016/02/16/reforme-de-1-orthographe-1-academiecomptait-bien-faire-appliquer-ses-rectifications-en-1990_4866505_4355770.html).

5. Selon une étude de l'Organisation de coopération et de développement économiques (OCDE), Littéracie, économie et société (1995), la proportion de personnes de 16 à 65 ans éprouvant des difficultés pour l'écriture, la lecture, ou l'utilisation des informations serait de 40,1 \% en France, taux qui place ce pays à l'avantdernière position (juste avant la Pologne) parmi les pays européens (https://fr.wikipedia.org/wiki/Illettrisme) [une autre étude date de 2000: http://www.oecd.org/fr/edu/innovation-education/39438013.pdf]. D'après l'Institut national de la statistique et des études économiques (Insee - 2011), $16 \%$ des personnes de 18 à 65 ans ont des difficultés dans les domaines fondamentaux de l'écrit en France (http://www.lefigaro.fr/ actualite-france/2014/09/18/01016-20140918ARTFIG00135-1-illettrisme-une-realite-dont-on-n-osepas-parler.php). 
(Adam, 2015: 31-32) et le texte est observé de deux points de vue compatibles: «le texte comme tout cohésif et le texte comme produit d'une pratique culturelle» (Maingueneau, 2014: 77).

La notion de genre rejoint en partie celle de tradition discursive. Notamment, les conventions de l'écriture peuvent s'étudier à travers la notion théorique de tradition discursive (voir Kabatek et al., 2010: 251). La langue étant un phénomène social, toutes les productions linguistiques s'inscrivent dans une certaine tradition: quel que soit le genre textuel en question, les auteurs qui tendent à l'adopter et à le produire se réfèrent à un modèle déjà existant et utilisent, consciemment ou inconsciemment, des éléments linguistiques qui lui sont typiques. C'est pourquoi les différentes traditions discursives peuvent s'identifier par la présence et la fréquence des éléments linguistiques communs - vocabulaire, mais aussi traits grammaticaux - qui les composent.

Les textes appartiennent à des genres, mais à l'intérieur d'un même genre peuvent prendre forme différentes traditions discursives selon les conditions de production des textes; un genre peut se réaliser linguistiquement de façons différentes. Si les auteurs - de lettres par exemple - ont une connaissance imparfaite de l'écriture normative, ils auront tendance à se référer à des modèles textuels qui leur sont plus familiers. La réalisation du genre de la lettre «officielle» à une autorité en droit reflétera ainsi la tradition discursive reproduisant les traits de l'écriture qui sont actuellement dominants. Il y aura (au moins) deux traditions discursives réalisant le genre de la «lettre officielle de demande d'aide à un juriste»: la traditionnelle qui suit la norme conventionnelle de l'écrit, apprise à l'école et respectée, entre autres, dans les journaux traditionnels comme Le Monde, et la «nouvelle» que l'on trouve sur Internet et qui suit d'autres principes d'écriture. Il convient de mentionner que l'écrit numérique est, naturellement, un vaste champ d'études qui est en train d'être exploré par des chercheurs représentant différentes disciplines. Rappelons, à titre d'exemple, les travaux présentés dans les ouvrages de référence de Herring et al. (2013), de Barats (2013), de Marcoccia (2016) et de Paveau (2017). Les caractéristiques de l'écrit numérique en soi ne sont cependant pas ce qui nous intéresse dans le cadre de cet article.

Dans l'étude de ces lettres, nous nous demanderons dans quelle mesure le genre est respecté et nous choisirons comme trait grammatical les techniques de jonction des propositions, qui sont définies comme étant tous les moyens qui relient les propositions entre elles (Raible, 1992 et 2001) ${ }^{6}$. Selon notre hypothèse, les textes produits par des non-professionnels de l'écriture correspondent moins parfaitement aux normes du français écrit que ceux de professionnels. De plus, ils sont potentiellement plus influencés par la langue parlée, la langue écrite jouant

6. Les jonctions interpropositionnelles ont été largement étudiées dans le contexte de l'acquisition du français comme langue seconde (voir par exemple les travaux de Welcomme [2012] et Buysse [2016a; 2016b], et leurs bibliographies). Cependant, à notre connaissance, les connexions entre propositions dans le discours diffusé par ordinateur n'ont pas fait l'objet d'études jusqu'à présent. 


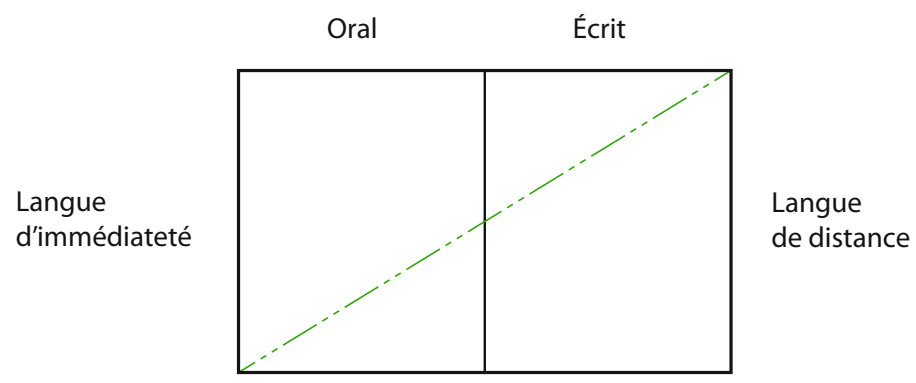

Figure 1

un rôle moindre dans le quotidien de ces locuteurs. Nous avançons donc qu'un tel corpus de lettres se situe à la charnière des textes écrits conventionnels et des textes oralisants, étant donné qu'elles sont écrites dans un but pratique par des non-spécialistes, sur un support électronique et souvent rapidement, mais avec la recherche d'un style convenant à un spécialiste.

Il est cependant clair que la distinction entre le caractère oral ou écrit de l'écriture n'est pas tranchée, mais se situe plutôt sur un continuum. Depuis Koch et Oesterreicher $(1985 ; 1994: 590$ et passim; 2011; voir aussi Koch, 1995), on fait la distinction entre la langue d'immédiateté (Sprache der Nähe) et la langue de distance (Sprache der Distanz). La figure 1 ci-dessus est adaptée à partir des travaux de Koch et Oesterreicher; le trait pointillé indique qu'il s'agit d'un continuum et non d'une dichotomie étanche.

L'immédiateté est caractéristique des textes oraux, mais les textes écrits peuvent aussi en présenter certains aspects, tels les messages entre amis ou d'autres écrits de tous les jours; en revanche, la langue de la distance, telle qu'elle est vue par Koch et Oesterreicher, est typique des textes écrits, mais certains textes oraux portent des caractéristiques de distance, comme les discours solennels. Pour mentionner un exemple de travaux dans la même lignée d'idées, Biber $(1995$; 2006) a montré que ce continuum constitue un facteur déterminant concernant le choix des divers éléments textuels.

Raible $(1992 ; 2001)$ discute les traits linguistiques liés à la langue d'immédiateté et à celle de distance quand il parle de l'agrégation et de l'intégration. En ce qui concerne la cohésion phrastique, et plus précisément les manières de faire la jonction des propositions utilisées dans un texte, les pôles du continuum sont: l'agrégation, contenant des caractéristiques de l'oralité (juxtapositions entre autres), et l'intégration, caractérisée par des propriétés du discours écrit élaboré, c'est-à-dire des techniques de condensation, des nominalisations, etc.

Chaque langue dispose d'une série de moyens textuels qui articulent un texte et réalisent la jonction des propositions. Dans une langue fortement caractérisée 
par la littératie, les jonctions interpropositionnelles ont tendance à se situer près du pôle d'intégration, alors que la langue orale préfère les jonctions proches du pôle d'agrégation.

Si l'intégration et l'agrégation sont des notions qui s'appliquent dans le domaine de la syntaxe phrastique, dans cette étude nous utilisons également la dichotomie densité/desserrement qui, en plus du degré d'intégration des jonctions, tient compte d'autres types d'éléments énonciatifs. Nous y reviendrons dans la section 5 .

La discussion autour de la densité ne devrait pas non plus négliger l'aspect du changement dans le temps. Il a été montré dans une perspective diachronique (voir Kabatek et al., 2010 et les références qui y sont données) que lorsqu'une langue écrite émerge, celle-ci a tendance à devenir textuellement plus complexe et plus intégrative avec le temps. Ceci a eu lieu, entre autres, en Espagne au Moyen Âge, lorsque la langue écrite était jeune, et en Suisse à partir du XIX ${ }^{e}$ siècle (presse rhéto-romane) (Kabatek et al., 2010). Serait-il possible que la langue écrite soit actuellement en train d'évoluer dans l'autre sens? Serait-elle en train de se décomplexifier et de devenir plus agrégative? Si c'était le cas, nous pourrions dire que nous sommes en train d'assister à une profonde transformation de la culture écrite, qui ne se verrait pas uniquement dans les textes produits par tout un chacun, mais aussi dans les écrits des professionnels de l'écriture. Il serait question d'une transformation de l'esthétique de l'écriture.

Dans cet article, la question de recherche est donc la suivante: dans les lettres écrites à des professionnels du droit par des locuteurs qui ne connaissent pas forcément bien le genre «attendu», quelles sont les caractéristiques du texte, notamment en ce qui concerne les jonctions interpropositionnelles? La réponse apportée nous permettra de poursuivre notre réflexion autour de la littératie et des conventions écrites en changement.

\section{Corpus}

Le corpus, recueilli en février 2016, contient 50 lettres de demande d'information destinées à des professionnels du droit et provenant du forum d'aide juridique gratuit http://www.juridissimo.com. Ont été prélevées les 25 premières lettres figurant sur deux chaînes de questions choisies au hasard, intitulées «Droit civil et familial» et «Droits du consommateur». Les échantillons de lettres contiennent respectivement 4130 et 4956 mots.

Il s'agit d'un site ouvert au public, et la lecture des lettres ne nécessite pas que l'on s'enregistre sur le site (bien que les auteurs des lettres le fassent). L'intérêt de la recherche - et des exemples de cet article - porte sur les choix linguistiques des lettres, pas sur les récits ou la vie de leurs auteurs. Toutefois, dans le souci de protection de l'identité des auteurs, les lettres ont été anonymisées.

À part l'anonymisation, les lettres ont été relevées telles quelles, sans aucune modification de l'orthographe ou de la ponctuation. Le respect des règles de 
l'orthographe varie: certaines lettres suivent les règles, d'autres beaucoup moins. L'emploi des majuscules est également variable. Une division en phrases (numérotées) a été faite pour les besoins de l'analyse selon la ponctuation existante et les alinéas: une séquence séparée par un point ou un alinéa par l'auteur de la lettre a été considérée comme une phrase.

Les lettres du corpus ont été analysées dans leur totalité, mais les trois premières - sélectionnées indépendamment de leurs caractéristiques - sont reproduites à la fin de cette section à titre d'exemple.

Ce qui est notable avec presque toutes les lettres du corpus, c'est qu'elles semblent avoir été rédigées ou mises en ligne rapidement: le temps entre l'inscription au site de leur auteur et l'envoi de la lettre n'est souvent que de quelques minutes (5, 10 et 15 minutes pour les lettres reproduites dans cette section); ces lettres ont pu être écrites au préalable à l'ordinateur - on ne peut donc pas savoir si elles ont effectivement été rédigées aussi vite. Mais c'est le produit fini qui nous intéresse ici, et nous ne cherchons pas à réfléchir à l'impact de la rapidité éventuelle de la rédaction.

Ces lettres représentent des spécimens de discours écrit réels; leur langue ne suit pas forcément la norme grammaticale du «bon usage». Elle a aussi parfois subi des influences de l'oral. Cependant, les lettres sont dans la forme écrite et il s'agit bien d'une forme de langue écrite. Une approche syntaxique et grammaticale traditionnelle adaptée à la langue écrite ne sera toutefois pas toujours suffisante en raison des écarts par rapport à la norme.

Généralement, les phrases de ces lettres se constituent d'unités syntaxiques de la langue écrite conventionnelle: nous y trouvons des syntagmes nominaux (SN) et des syntagmes verbaux (SV) qui constituent des propositions traditionnelles, dont la forme canonique minimale se réduit à un SN sujet et un SV (Riegel et al., 2009: 240 et 784-785 ${ }^{7}$. La proposition sera considérée comme l'unité de base de l'analyse. Ces propositions peuvent ensuite être liées les unes aux autres par différents types de jonctions pour former des phrases complexes.

Nous avons relevé un petit nombre de séquences qui ne remplissent pas les critères mentionnés de proposition, parce qu'il y manque une partie constituante (soit un SV, soit un SN, soit les deux) (ex. «donc plus de pension (normal)» $[1 ; 3]^{8}$; «De même pour 1 or rose» $[2 ; 5]$ ). Sémantiquement, ces séquences ${ }^{9}$ expriment cependant une prédication et sont interprétables de la même manière que les propositions, et elles sont aussi reliées au reste des énoncés par les mêmes types de relations de dépendance que les propositions syntaxiques. C'est pourquoi nous les assimilerons

7. Un éventuel subordonnant ou connecteur, généralement placé en tête, peut faire partie de ces séquences.

8. Le numéro entre parenthèses (ou crochets ici) identifie le passage du corpus cité comme suit: échantillon de lettres 1 ou 2 ; numéro de la lettre (1-25).

9. Ces unités minimales, qui peuvent former des énoncés autonomes mais qui ne prennent pas appui directement sur les fonctions et les catégories grammaticales, sont appelées «noyaux» dans la macrosyntaxe élaborée pour l'étude de la langue parlée (Blanche-Benveniste, 1990: 114; 2010: 125; voir aussi Berrendonner, 1990). 
à des propositions dans l'analyse (pour les phrases averbales, voir Lefeuvre [1999]). Le nombre total des propositions s'élève à 876 , dont 643 avec verbe fini $(73,4 \%)$, 79 avec verbe non fini $(9 \%)$ et 154 sans verbe ${ }^{10}(17,6 \%)$. Pour leur part, les jonctions sont au nombre de 826 dans le corpus (la première proposition de chacune des 50 lettres ne pouvant entretenir de relation de jonction avec ce qui précède).

Les trois lettres ici reproduites intégralement contiennent respectivement 148 mots, 47 mots et 292 mots (la longueur moyenne des lettres du corpus est de 182 mots).

Lettre 1:

\section{Bonjour}

(1) [PRÉNOM] j'ai 53 ans, il y a 20 ans mes parent m'ont donné un terrain et avec mon épouse nous y avons construit une maison. (2) nous étions mariés sous contrat de mariage et la donation était uniquement à mon nom. (3) Il y a 8 ans nous avons divorcé j'ai gardé la maison et règle le crédit tous les mois. (4) Le jugement de divorce est prononcé je dois une «récompense» à mon épouse mais nous ne tombons pas d'accord car elle veut la moitié de la vente. (5) Elle veut faire traîner la situation car elle sait que je ne vis plus dans la maison, que j'ai refait ma vie et que cette maison est une charge énorme pour moi ...

(6) Ma question est simple! (7) Ai je le droit de louer ma maison et de garder les loyer pour payer la charge de cette dernière?

Lettre 2:

(1) Mon fils a été retiré y a 6 mois je me suis mariée y a 2 mois la juge des enfants etait au courant mais a laissé mon nom de jeune fille est ce que cela peut constitué une erreur de procédure? (2) merci de votre réponse $\hat{\theta}$

Lettre 3:

(1) voila mon ex-mari versait jusqu'en janvier dernier 200 euros de pension alimentaire pour mes 2 filles âgées de 21 et 18 ans ma fille de 18 ans a quitté le domicile pour s'installer avec son compagnon et leur enfant donc plus de pension (normal) mais en ce qui concerne ma fille de 21 ans il a coupé net de sa propre initiative la pension due pour elle, en effet le jugement stipule qu'au dela de la majorité de l'enfant tant que celui-ci ne peut subvenir a ses besoins seul et qu'il vit a mon domicile la pension est due.

(2) Je suis allée voir un huissier de justice sur les conseils de la maison de justice et celui-ci m'a bien confirmé qu'il etait en tort il a pris mes papiers et les renseignements. (3) Seulement mon ex a dit au téléphone ( je l'avais joint en m'apercecevant que je navais pas eu le virement) il m'a dit si elle a besoin de quelque chose (de l'argent elle me demande!) le lendemain il avait versé sur le compte de ma fille (compte qui est ouvert au cas ou elle trouve un emploi) la somme de 100 euros ma question est: dois-je considérer que cela est la pension? (4) je crois que non et ma fille et

10. Environ deux tiers des propositions sans verbe sont des formules de début ou de fin de lettre (bonjour, cordialement, etc.). 
moi sommes unanimes nous ne voulons pas y toucher on ne sait jamais. (5) je suis au rsa je touche pour 2 la somme de 522 euros je paye tous les frais de ma fille. (6) merci de me sortir de cette situation qui me fiche la vie en l'air de plus étant tellement en colère et angoissée j’ai envoyé un message a mon ex lui disant que j'avais pris des renseignements auprès d'un huissier de justice quelle gourde non?

\section{Organisation du texte}

Pour analyser la façon dont les lettres s'organisent du point de vue textuel, nous aborderons deux facettes différentes du corpus. La cohérence des lettres et le genre qu'elles représentent seront analysés dans la section 4.1. La section 4.2 se concentrera sur la cohésion des lettres, et cela surtout par les jonctions qui y figurent. Nous suivons la distinction habituelle entre cohérence et cohésion (voir Halliday et Hasan, 1976; Charaudeau et Maingueneau, 2002 : 99) : la cohérence relève de l'organisation logicosémantique du texte, alors que par cohésion, on entend les moyens linguistiques et typographiques qui contribuent à créer de la cohérence.

\subsection{Cohérence des lettres: respect du genre}

Il est bien connu (voir Luzzati et Luzzati, 1987: 18) que le discours écrit conventionnel, sous sa forme prototypique, doit être cohérent et nécessite une planification, alors que le discours oral, régi par des critères énonciatifs, peut se caractériser par une linéarité textuelle. Les lettres rédigées dans l'intention d'obtenir une aide de la part d'un juriste représentent un genre écrit dont il est logique d'attendre planification et cohérence. L'examen de nos lettres révèle que cela a effectivement été le cas: malgré leur ponctuation défaillante et les jonctions parfois peu habituelles entre les propositions (voir la section 4.2 ci-dessous), ces écrits font tout de même clairement preuve d'une planification et ne suivent donc pas les habitudes du discours oral familier. Ainsi est-il possible de relever dans ces lettres une certaine macrostructure récurrente ou organisation de cinq unités rhétoriques (terme de Matthiessen et Thompson, 2011: 287), ces dernières remplissant chacune un certain but: une formule de commencement avec des informations éventuelles sur l'auteur et/ou préparation de l'entrée en matière (DÉBUT), la description d'une situation (FOND), la présentation d'un problème (PROBLÈME), une demande d'aide adressée au juriste (QUESTION) et, pour finir, une formule de clôture avec remerciements éventuels (FIN). À l'intérieur de ces parties et entre elles, les propositions sont organisées selon une certaine logique (microstructure). En ce sens, les lettres remplissent le genre de la lettre de demande d'aide juridique auquel elles tendent: elles ont toutes été envoyées à leur destinataire dans le but d'obtenir une réponse à un problème et en tant que telles ont été acceptées par le modérateur du site ${ }^{11}$.

11. Pour Matthiessen et Thompson (2011: 287), le critère de fonctionnalité est à lui seul suffisant: un texte qui remplit sa fonction est par définition cohérent, indépendamment de la manière dont cette cohérence est réalisée dans la pratique. 
1. DÉBuT
[proposition] J [proposition]

2. FOND

J [proposition] J [proposition] J [proposition] J [proposition] J [proposition] [...]

3. PROBLÈME

$\mathrm{J}$ [proposition] J [proposition] J [proposition] J [proposition] [...]

4. QUESTION

J [proposition] J [proposition] J [proposition] [...]

5. FIN

J [proposition] J [proposition]

Figure 2 - Macro- et microstructure des lettres adressées aux juristes du forum.

Dans chaque case, il peut y avoir une, deux ou plusieurs propositions

La figure 2 ci-dessus présente le niveau de macro- et de microstructure des lettres. La macrostructure se constitue des cinq parties numérotées, qui à leur tour se composent de propositions liées entre elles par différents types de jonctions (la microstructure). Les jonctions, marquées dans les lettres de manière explicite ou implicite, sont indiquées par «J».

Voici un exemple des unités de macrostructure (la lettre est reproduite dans sa totalité):

[1] [DÉBUT] Bonjour, [alinéa] [FOND] J ai vendu un bien et l acheteur $m$ as fait 4cheques . [alinéa] [PROBLĖME] Seul 2 on été réglés, [Question] combien de temps ais je pour intenter une action en justice? [alinéa] Quel est le délai de prescription ou forclusion pour ces chèques? [alinéa] [FIN] Cordialement.

$(2 ; 16)$

Les unités 1 et/ou 5 sont parfois omises, mais les trois unités du milieu sont repérables dans pratiquement chacune des lettres. Seules deux lettres du corpus attaquent directement le PROBLÈME sans exposer le FOND. Voici une de ces lettres:

[2] [DÉBuT] bonjour, [alinéa] je ne m'adresse peu etre pas au bon endroit; Me voila face a un soucis: [PROBLÈME] je reçoit pas loin de 10 fois par semaine (voir plus) un appel d'un numéro venant du sud est de la france qui sonne jusqu'a se qu'on décroche et raccroche aussitot . [alinéa] [QUESTION] que puis je faire juridiquement? cela devient soulant [alinéa] [FIN] merci

$(2 ; 7)$

Il arrive qu'une unité rhétorique ne s'en tienne pas qu'à son information centrale, mais qu'elle soit étoffée par des informations supplémentaires. Ainsi, lors de la QUESTION ou de l'exposition du PROBLÈME, l'auteur fait parfois allusion 
à ses sentiments ou à son comportement vis-à-vis de sa situation, comme dans l'exemple [2] ci-dessus à la fin de la QUESTION («cela devient soulant»), ou dans l'exemple suivant (les passages relevant du commentaire personnel sont indiqués en italique par les auteurs de cet article):

[3] [...] [Question] est- il possible de payer et ensuite contester en saisissant la justice? ( je pose cette question pour différentes raisons.) [alinéa] (Ps: je ne peux bénéficier de l'aide juridique car je dépasse un peu le plafond, et j' ai déjà demandé les honoraires à un avocat mais c' est assez coûteux avec tous ce qu' il me reste à payer) [alinéa] [FIN] Je vous remercie. [alinéa] Cordialement.

$(2 ; 22)$

Dans deux autres lettres, l'auteur anticipe la réponse possible du juriste, voir l'exemple [4] ci-dessous:

[4] [...] [Question] Comment puis-je prétende à l'héritage? [alinéa] Quelles sont les procédures que je dois entamer. [...] Je pense que la femme de mon père lui a fait changer son testament. Et qu'étant non reconnu, je n'ai le droit à rien. [...]

$(1 ; 7)$

Plus fréquemment, l'auteur revient sur des explications concernant le fond lors du PROBLÈmE ou de la QUESTION. Dans l'exemple suivant, l'auteur avait déjà mis en avant les détails pratiques liés au problème du changement d'école de ses enfants. L'élaboration de la fin de la lettre (en italique) ne fait que réexpliquer le problème du point de vue du père de ses enfants:

[5] [...] [Question] Dans quelles mesures ai-je le droit de refuser ou pas? selon la loi, et le fait d'avoir la garde des enfants restant majoritaire, suis-je dans mon droit si je les change de départements? [alinéa] Tout cela en sachant qu'aujourd'bui je fais $18 \mathrm{~km}$ pour emmener mes enfants à l'école et que dans le cas ou ils changent de département c'est leur père qui devra les faire et qu'il ne veut pas changer ses jours ou son mode de garde définitivement.

$(1 ; 9)$

Nous avons vu jusqu’à présent que ces lettres - malgré le non-respect partiel des conventions linguistiques - présentent une macrostructure claire qui leur permet d'exposer un message de manière cohérente et d'atteindre leur but. De cette façon, elles respectent le genre voulu et représentent simplement une tradition discursive nouvelle par rapport à la lettre traditionnelle écrite à la main. La macrostructure est donc un élément stabilisant les écarts par rapport à la norme orthographique et grammaticale. De la tradition discursive de la lettre traditionnelle, ces textes gardent aussi, entre autres, une recherche lexicale correspondant traditionnellement au genre en question (par exemple, dans les trois lettres présentées intégralement dans la section $3:$ «le jugement de divorce est prononcé»; «constituer une erreur de procédure»; «quitter le domicile»...). 


\subsection{Cohésion des lettres: jonctions}

Dans cette section, on s'intéressera à la microstructure des lettres et à la cohésion qui en ressort à travers l'examen des jonctions ${ }^{12}$ interpropositionnelles (Raible, 1992 et 2001). Nous chercherons également à mesurer le degré de densité de ces textes en définissant les relations logiques entre leurs propositions et en les mettant en rapport avec les techniques de jonction qui y sont utilisées ${ }^{13}$, aspect de la textualité qui ressort clairement dans notre corpus comme obéissant à des règles autres que celles de l'écriture conventionnelle.

Dans la théorie de Raible (1992 et 2001), les jonctions - ou l'ensemble des moyens qui relient les propositions entre elles - peuvent former des liens plus ou moins serrés, situant les propositions sur un continuum dont les deux pôles extrêmes sont l'entière agrégation (absence de marquage linguistique de jonction entre deux propositions) et l'intégration (fusion complète des propositions) (Raible, 1992: 303). Pour déterminer la nature de la jonction entre deux propositions, sont pris en compte deux facteurs qui agissent simultanément: la présence d'un joncteur explicite éventuel (conjonction, connecteur ou préposition) et un moyen syntaxique, le degré de dépendance entre les propositions (juxtaposition, coordination, subordination). Les joncteurs occupent un rôle majeur dans cette représentation: ils ne sont régulièrement absents que dans le cas d'agrégation extrême (I) et d'intégration extrême (VII). Le tableau 1 expose la classification des jonctions présentée par Raible (1992: 303) ${ }^{14}$, illustrée par des exemples tirés de notre corpus où les joncteurs sont soulignés.

Le continuum proposé par Raible rend compte des cas de figure de la langue écrite conventionnelle qui suit la norme grammaticale et celle de la ponctuation. Raible ne semble pas se prononcer explicitement sur la ponctuation, mais quand on analyse la langue écrite, un choix de signes de ponctuation divergent peut déjà, en soi, indiquer un degré d'intégration différent; la ponctuation contribue naturellement à la segmentation du texte écrit mais également à son organisation syntaxique (voir Gautier, 2014: 28). Le modèle de Raible demande à être précisé tout particulièrement sur la ponctuation.

12. Suivant Matthiessen et Thompson (2011: 276-284), nous considérons que toutes les propositions appelées subordonnées dans la tradition grammaticale (par ex. Riegel et al., 2009: 780-781, 785) ne constituent pas des cas de combinaisons logiques ou circonstancielles de propositions. Les cas où la subordonnée dépend d'un constituant de la proposition principale et forme un complément ou un modifieur à l'intérieur d'un syntagme verbal ou nominal, sont conditionnés par des contraintes grammaticales et n'expriment pas (ou peu) des liens logiques. Comme l'intérêt de cette étude porte sur les liens logiques s'établissant entre les propositions, seront prises en compte uniquement celles qui sont directement dominées par le niveau phrase $(\mathrm{S})$, de même que les différentes phrases des textes.

13. Notons cependant que la textualité se compose de plusieurs éléments dont la jonction ne représente qu'une seule partie. Par exemple, Adam (2015: chap. 3) fait l'inventaire des différents types de liages textuels, à partir des références anaphoriques jusqu'aux connecteurs en passant par l'ellipse.

14. Nous avons unifié les catégories VI et VII de Raible pour lesquelles il fait une différence entre les syntagmes régis par une préposition complexe, d'une part, ou simple, d'autre part. Par conséquent, notre catégorie VII correspond à la catégorie VIII dans son étude. 


\begin{tabular}{|l|l|l|}
\hline I & $\begin{array}{l}\text { Juxtaposition de deux phrases; } \\
\text { pas de joncteur }\end{array}$ & $\begin{array}{l}\text { «Je suis pacsé avec une allemande. } \\
\text { Nous vivons à Lille.» }(1 ; 4)\end{array}$ \\
\hline II & $\begin{array}{l}\text { Deux phrases séparées; joncteur qui } \\
\text { renvoie anaphoriquement à la phrase } \\
\text { précédente (ou à une partie de celle-ci) }\end{array}$ & $\begin{array}{l}\text { «...] or financièrement je ne pourrais } \\
\text { pas m'en sortir sans ça. [alinéa] C'est } \\
\text { pour cela que quand je lui ai dit que } \\
\text { je refusais }[\ldots] » ~\end{array}$ \\
\hline III & $\begin{array}{l}\text { Deux propositions coordonnées; } \\
\text { présence de joncteur }\end{array}$ & $\begin{array}{l}\text { «Je viens vers vous car j'ai un problème» } \\
(2 ; 2)\end{array}$ \\
\hline IV & $\begin{array}{l}\text { Deux propositions dont l'une est } \\
\text { subordonnée à l'autre; présence de } \\
\text { joncteur }\end{array}$ & $\begin{array}{l}\text { «Si ce n'est pas le cas que puis je mettre } \\
\text { dans cette clause?» }(1 ; 11)\end{array}$ \\
\hline V & $\begin{array}{l}\text { Deux propositions dont l'une est } \\
\text { subordonnée à l'autre et réduite à } \\
\text { l'infinitif/ au participe/ au gérondif; } \\
\text { présence éventuelle de joncteur }\end{array}$ & $\begin{array}{l}\text { «je me suis inscrit en octobre } \\
\text { à une école en ligne pour passer } \\
\text { un cap coiffure en ligne» }(2 ; 2)\end{array}$ \\
\hline VI & $\begin{array}{l}\text { Une des propositions est réduite à une } \\
\text { nominalisation; présence de joncteur }\end{array}$ & $\begin{array}{l}\text { «Malgré les plaintes de nos voisins } \\
\text { concernant le bruit ils ont refusé de } \\
\text { baisser le volume sonore.» }(1 ; 21)\end{array}$ \\
\hline VII & $\begin{array}{l}\text { La seconde proposition est devenue } \\
\text { un actant du verbe de la première } \\
\text { proposition; pas de joncteur }\end{array}$ & $\begin{array}{l}\text { «je souhaiterais pour ma part } \\
\text { une garde alternée» }(1 ; 4)\end{array}$ \\
\hline
\end{tabular}

Tableau 1 - Jonctions d'après Raible, de l'agrégation à l'intégration (I-VII)

Le tableau 2 présente la situation de notre corpus. Il est organisé suivant le modèle de Raible en fonction d'un axe croissant de dépendance interpropositionnelle (cf. tableau 1). Le tableau commence par la situation où les phrases sont entièrement indépendantes (catégorie I-a: deux phrases séparées par un point, sans joncteur) et aboutit à celle où deux propositions sont totalement unies pour ne former qu'une seule phrase simple (la deuxième étant devenue un constituant de la première, catégorie VII).

La question se posera de savoir si cet ordre de «nominalité» croissante, ou de «verbalité» décroissante (Lehmann, 2011: 193-200), où l'une des phrases perd de plus en plus de sa "phrasticité» ${ }^{15}$ au fur et à mesure que l'on descend dans le tableau, reflète aussi de manière fiable la densité du texte. Nous y reviendrons.

La jonction peut se faire soit de manière explicite à l'aide de joncteurs ou de la ponctuation, mais aussi de manière implicite, sans élément linguistique ou typographique apparent. Dans les quatre premières catégories du tableau 2, la jonction se compose de trois facteurs au lieu de deux: emploi d'un joncteur, ponctuation et degré de dépendance interpropositionnelle. Si l'on compare ce tableau avec celui de Raible, on constate d'emblée que le degré d'intégration dépend aussi de la ponctuation.

15. Le terme employé par Lehmann $(2011: 193)$ est «desententialization». 
Une remarque reste à faire, liée au caractère écrit du corpus. Si nous tenons compte de la ponctuation, s'ensuit-il que l'usage des majuscules demande aussi une attention particulière, surtout s'il ne suit pas les règles du «bon usage»? Lors de l'analyse du corpus, nous avons parfois noté un emploi peu canonique, mais certains appareils ou logiciels ajoutant automatiquement des majuscules après un alinéa ou un point, il ne faut peut-être pas trop prêter attention à des usages peu conventionnels de majuscules. Il ne s'agit pas toujours forcément d'un choix conscient de l'auteur du texte.

Voici ci-dessous le tableau 2 fait à partir de notre corpus. Il sera suivi de commentaires de chacune de ses catégories, avec une attention particulière apportée à la ponctuation.

\begin{tabular}{|c|c|c|c|}
\hline Catégorie & Type de jonction & Exemples & $\begin{array}{l}\text { Nombre et } \\
\text { pourcentage } \\
{[\text { total }=826} \\
(100 \%)]\end{array}$ \\
\hline $\mathrm{I}-\mathrm{a}$ & $\begin{array}{l}\text { Juxtaposition de deux } \\
\text { phrases; pas de joncteur } \\
\text { POINT/ALINÉA }{ }^{16}\end{array}$ & $\begin{array}{l}\text { «Je suis pacsé avec une } \\
\text { allemande. Nous vivons } \\
\text { à Lille.» }(1 ; 4) \\
\text { «Nous avons été alerté } \\
\text { par nos voisins. Une } \\
\text { véritable soirée organisée } \\
\text { avec traiteurs, dj, lumières } \\
\text { etc. Env } 60 \text { personnes } \\
\text { présentes.» }(1 ; 21)\end{array}$ & $403(48,8 \%)$ \\
\hline $\mathrm{I}-\mathrm{b}$ & $\begin{array}{l}\text { Juxtaposition de deux } \\
\text { propositions; } \\
\text { pas de joncteur } \\
\text { VIRGULE }\end{array}$ & $\begin{array}{l}\text { «Les enfants sont déjà au } \\
\text { courant, mon fils de } 13 \text { ans } \\
\text { souhaiterait rester avec sa } \\
\text { mère» }(1 ; 13)\end{array}$ & $57(6,9 \%)$ \\
\hline $\mathrm{I}-\mathrm{c}$ & $\begin{array}{l}\text { Juxtaposition de deux } \\
\text { propositions; } \\
\text { pas de joncteur } \\
\text { PAS DE PONCTUATION }\end{array}$ & $\begin{array}{l}\text { «La garde alternée n'est } \\
\text { donc plus possible je vais } \\
\text { devoir demander la garde } \\
\text { de ma fille» }(1 ; 10)\end{array}$ & $50(6,1 \%)$ \\
\hline II-a & $\begin{array}{l}\text { Deux phrases séparées; } \\
\text { joncteur qui renvoie } \\
\text { anaphoriquement à la phrase } \\
\text { précédente (ou à une partie) } \\
\text { POINT/ALINÉA }\end{array}$ & $\begin{array}{l}\text { «[...] or financièrement je } \\
\text { ne pourrais pas m'en sortir } \\
\text { sans ça. [alinéa] C'est pour } \\
\text { cela que quand je lui ai dit } \\
\text { que je refusais }[\ldots] »(1 ; 6)\end{array}$ & $1(0,1 \%)$ \\
\hline
\end{tabular}

16. Le point d'exclamation ou d'interrogation, le point-virgule, les deux-points et les points de suspension ainsi que les parenthèses sont considérés ici comme des points. 


\begin{tabular}{|c|c|c|c|}
\hline II-b & $\begin{array}{l}\text { Deux propositions séparées; } \\
\text { joncteur qui renvoie } \\
\text { anaphoriquement à la phrase } \\
\text { précédente (ou à une partie) } \\
\text { VIRGULE }\end{array}$ & - & - \\
\hline II-c & $\begin{array}{l}\text { Deux propositions séparées; } \\
\text { joncteur qui renvoie } \\
\text { anaphoriquement à la } \\
\text { proposition précédente } \\
\text { (ou à une partie) } \\
\text { PAS DE PONCTUATION }\end{array}$ & - & - \\
\hline III-a & $\begin{array}{l}\text { Deux phrases coordonnées; } \\
\text { présence de joncteur } \\
\text { POINT/ALINÉA }\end{array}$ & $\begin{array}{l}\text { «j'ai signée un bail pour } \\
\text { un emplacement publicitaire } \\
\text { le } 27 \text { janvier } 2016 \text { à mon } \\
\text { domicile. Or samedi il } 1 \text { a } \\
\text { installé alors que je suis } \\
\text { encore dans mon délai de } \\
\text { réflexion.» }(2 ; 13)\end{array}$ & $38(4,6 \%)$ \\
\hline III-b & $\begin{array}{l}\text { Deux propositions } \\
\text { coordonnées; présence } \\
\text { de joncteur } \\
\text { VIRGULE }\end{array}$ & $\begin{array}{l}\text { «Qu'il a fait changer les } \\
\text { bougies....les injecteurs.... } \\
\text { ect..et qu'il a fait } 1000 \mathrm{kms} \\
\text { depuis l'achat ,Et que la ....la } \\
\text { moto est partis pour passer } \\
\text { à la valise.» }(2 ; 3)\end{array}$ & $25(3,0 \%)$ \\
\hline III-c & $\begin{array}{l}\text { Deux propositions } \\
\text { coordonnées; présence } \\
\text { de joncteur } \\
\text { PAS DE PONCTUATION }\end{array}$ & $\begin{array}{l}\text { "Je viens vers vous car j'ai un } \\
\text { problème» }(2 ; 2)\end{array}$ & $135(16,3 \%)$ \\
\hline IV-a & $\begin{array}{l}\text { Deux propositions, dont } \\
\text { l'une est subordonnée à } \\
\text { l'autre; présence de joncteur } \\
\text { POINT/ALINÉA }\end{array}$ & $\begin{array}{l}\text { «Sachant que mon père était } \\
\text { devenu sénile (dernière fois } \\
\text { au téléphone en } 2008 \text {, il avait } \\
\text { eu du mal à me reconnaître, } \\
\text { du moins au début) [alinéa] } \\
\text { Je pense que la femme de } \\
\text { mon père lui a fait changer } \\
\text { son testament.» }(1 ; 7)\end{array}$ & $9(1,1 \%)$ \\
\hline IV-b & $\begin{array}{l}\text { Deux propositions, dont } \\
\text { l'une est subordonnée à } \\
\text { l'autre; présence de joncteur } \\
\text { VIRGULE }\end{array}$ & $\begin{array}{l}\text { «Etant donné que je reste } \\
\text { au domicile conjugal, J’ai } \\
\text { peur qu'elle puisse me } \\
\text { réclamer une contribution } \\
\text { financière» }(1 ; 11)\end{array}$ & $9(1,1 \%)$ \\
\hline IV-c & $\begin{array}{l}\text { Deux propositions, dont } \\
\text { l'une est subordonnée à } \\
\text { l'autre; présence de joncteur } \\
\text { PAS DE PONCTUATION }\end{array}$ & $\begin{array}{l}\text { «Si ce n'est pas le cas que } \\
\text { puis je mettre dans cette } \\
\text { clause?» }(1 ; 11)\end{array}$ & $34(4,1 \%)$ \\
\hline
\end{tabular}




\begin{tabular}{|c|c|c|c|}
\hline$V-a$ & $\begin{array}{l}\text { Deux propositions, dont } \\
\text { l'une est subordonnée à } \\
\text { l'autre et réduite à une } \\
\text { forme verbale non finie; } \\
\text { pas de joncteur }\end{array}$ & $\begin{array}{l}\text { «La garde des enfants étant } \\
\text { partagée chacun des époux } \\
\text { assumera sa partie financière» } \\
(1 ; 11)\end{array}$ & $23(2,8 \%)$ \\
\hline$V-b$ & $\begin{array}{l}\text { Deux propositions, dont } \\
\text { l'une est subordonnée à } \\
\text { l'autre et réduite à une forme } \\
\text { verbale non finie; présence } \\
\text { de joncteur }\end{array}$ & $\begin{array}{l}\text { «je me suis inscrit en octobre } \\
\text { à une école en ligne pour } \\
\text { passer un cap coiffure en } \\
\text { ligne» }(2 ; 2)\end{array}$ & $42(5,1 \%)$ \\
\hline VI & $\begin{array}{l}\text { Une des propositions } \\
\text { est réduite à une } \\
\text { nominalisation; } \\
\text { présence de joncteur }{ }^{17}\end{array}$ & $\begin{array}{l}\text { «Si celle-ci est vendue avant } \\
\text { la prononciation du divorce» } \\
(1 ; 5) \\
\text { «Malgré les plaintes de nos } \\
\text { voisins concernant le bruit } \\
\text { ils ont refusé de baisser le } \\
\text { volume sonore. }(1 ; 21)\end{array}$ & Pas de nombre \\
\hline VII & $\begin{array}{l}\text { La seconde proposition est } \\
\text { devenue un actant du verbe } \\
\text { de la première proposition; } \\
\text { pas de joncteur }{ }^{18}\end{array}$ & $\begin{array}{l}\text { «Je vous remercie par avance } \\
\text { pour vos réponses.» }(1 ; 21)\end{array}$ & Pas de nombre \\
\hline
\end{tabular}

Tableau 2 - Types de jonctions entre les propositions dans les lettres aux juristes

\subsubsection{Catégorie I: pôle agrégatif de l'axe}

La catégorie I, réunissant $61,7 \%$ de toutes les occurrences, est la plus importante du corpus ${ }^{19}$.

Un trait marquant dans nombre de ces lettres, qui les éloigne peut-être le plus de la norme écrite, est que les propositions à verbe fini peuvent être enchaînées les unes après les autres par la juxtaposition, sans joncteur ou signe de ponctuation (catégorie I-c). Ce type de jonction représente 50 occurrences $(6,1 \%)$ dans le corpus.

17. Cette catégorie a beaucoup d'intérêt théorique, mais elle est inopérante dans une étude empirique sur les jonctions, particulièrement s'il s'agit de données quantitatives. Les nominalisations trouvées dans le texte ne peuvent pas toutes être mises en parallèle avec des constructions verbales virtuelles. C'est pourquoi aucun nombre n'est donné dans la dernière colonne.

18. Cette catégorie a un intérêt théorique tout comme la catégorie VI, mais elle est inopérante, elle aussi, dans une étude empirique sur les jonctions: aucun nombre n'est donné dans la dernière colonne. Dans l'exemple du tableau, il y a la préposition pour: je vous remercie pour vos réponses. Dans la mesure où pour vos réponses est considéré comme complément du verbe remercier faisant partie de sa structure argumentale, la préposition pour n'a aucun rôle jonctif.

19. Notons toutefois que les formules de début et de fin de lettre, qui n'appartiennent pas pleinement au corps de la lettre, augmentent le nombre de la catégorie I-a d'une petite centaine d'occurrences. 
La tendance à omettre tout joncteur explicite ne figure pas chez tous les auteurs, ni de manière systématique. Elle semble apte à apparaître lorsque les propositions sont liées par une simple relation d'addition (exemple [6]) ou lors d'une relation d'addition qui exemplifie le sens de la proposition qui précède (exemple [7]), ou encore, plus rarement, lors d'une relation de conséquence (exemple [8]):

[6] Je me présente je viens d'Alsace plus exactement de richwiller petit village situé près $\mathrm{d}$ emulhouse $:-$ et jai 25 ans $(2 ; 2)$

[7] elle gagnent très bien son salaire est vers les 5000 EUROS , on habite pas ensemble $(1 ; 25)$

[8] La garde alternée n'est donc plus possible je vais devoir demander la garde de ma fille $(1 ; 10)$

La relation entre les propositions restant tout de même sous-déterminée à cause du manque d'un signe linguistique apparent, la nature de la relation logique peut rester ambiguë dans certains cas:

[9] Je ne suis pas égoïste je pense beaucoup au bien être de ma fille $(1 ; 10)$

S'agit-il ici d'une simple addition ou d'une conséquence exprimée dans la proposition de droite, ou d'une cause exprimée dans la proposition de gauche? Nous considérons que pour une interprétation causale, il faudrait un élément causal explicite. En son absence, on comprendra la proposition de droite comme la conséquence de ce qui a été dit précédemment, car la relation de conséquence suit directement le principe d'iconicité de la langue et serait donc moins marquée. Mais la proposition de droite pourrait aussi servir à exemplifier ce qui vient d'être dit, ou simplement s'y ajouter. L'intention de l'auteur ne ressort pas de manière explicite. La même remarque que pour la relation causale vaut pour plusieurs autres relations logiques qui nécessitent d'être marquées par un élément sémantique: le but, la concession, la condition, la restriction. Si cet élément sémantique manque dans la proposition, le lecteur n'a pas de moyen d'inférer la nature exacte de la relation sémantique qui s'instaure entre les propositions, et le message demeure opaque (sous-déterminé) ${ }^{20}$.

Dans tous les cas recensés, un point conviendrait entre les deux propositions; cependant la nature de la relation ne serait pas plus précisée alors. Simplement, ce serait suivre les règles typographiques: la langue écrite conventionnelle opterait pour une frontière claire entre les deux propositions.

20. Ceci correspond à l'ordre de complexité croissante des relations logiques tel que Raible (1992: 131-132) le présente. La relation de base entre deux états de choses est simplement qu'il y ait un lien quelconque; viennent ensuite la relation temporelle et la relation causale; la finalité et la concession les suivent. 
Mais comme nous l'avons dit, la succession des propositions sans signe de ponctuation répond toutefois à un principe d'iconicité dans la langue. Bien que non normatif, c'est probablement délibéré de la part des auteurs. Ceux-ci ont donc choisi d'exprimer les deux propositions comme intimement liées, sans signes de ponctuation ou autres marques typographiques. Mais ils ont aussi choisi de ne pas les intégrer entre elles par un quelconque moyen syntaxique. De plus, ils ont choisi de ne pas spécifier la relation sémantique qui s'instaure entre elles. Ce sont des marques d'une expression plus «lâche» que ne le serait le choix d'un auteur respectant les conventions de l'écrit.

Aux cas susmentionnés s'ajoute la catégorie I-b, où deux propositions sont juxtaposées à l'aide d'une virgule (sans mot de jonction). Cette catégorie est également bien représentée avec 57 occurrences (6,9\%). Ces cas se comportent de la même manière du point de vue de l'enchaînement logique des propositions, c'est-à-dire que la nature de la relation sémantique n'est pas spécifiée.

Le nombre des occurrences appartenant aux catégories I-b et I-c, manifestant un certain desserrement textuel, est remarquable (107, soit $13 \%$ en tout).

\subsubsection{Catégorie II: joncteur anaphorique}

Un trait particulier dans ce corpus est que les jonctions à l'aide de joncteurs renvoyant anaphoriquement à la phrase ou à une partie de la phrase précédente en sont presque entièrement absentes. Une seule occurrence a été recensée, qui est l'exemple du tableau («C'est pour cela que»). Une occurrence ne permet pas de tirer des conclusions poussées, mais il est possible qu'un joncteur anaphorique soit ressenti plus clairement comme un introducteur d'un nouveau début, ce qui encouragerait l'auteur à le faire précéder d'un point (au lieu d'une virgule ou d'une simple omission de ponctuation), contrairement à ce qui peut arriver lorsqu'aucun joncteur n'est présent (catégorie I) ou avec un joncteur non anaphorique (catégorie III).

Les joncteurs anaphoriques jouent un rôle double: ils déterminent à la fois le lien logique et renvoient à quelque chose dans le texte précédent. C'est pourquoi ils créent des liens plus serrés (denses) entre les propositions que les autres joncteurs, et leur absence contribue au desserrement du texte.

\subsubsection{Catégorie III: joncteur de coordination}

La catégorie III, où les propositions sont au même niveau syntaxique entre elles et un joncteur est présent, constitue la deuxième du point de vue de sa taille: elle rassemble 198 occurrences $(24 \%)$. Cette catégorie ne présente pas de particularités notables.

\subsubsection{Catégorie IV: joncteur de subordination}

La catégorie IV réunit 52 occurrences (6,3\%). La catégorie IV-a (9 occurrences), où une proposition introduite par un joncteur de subordination est séparée par un point, est un emploi qui s'écarte de la norme; on ne le relève qu'avec les joncteurs que et sachant que: 
[10] Sachant que l' acheteur a toujours su que je n'avais jamais vu en fonctionnement l'appareil $([\ldots])$ et qu'il a pris la responsabilité de l'acheter malgré tout.

$(2 ; 21)$

Ici, un joncteur révèle la présence d'un axe de transfert, et le point - qui coupe la continuité entre deux propositions - n'apparaît que six fois dans le corpus ${ }^{21}$, ce qui montre que certaines conventions de ponctuation sont respectées dans ces lettres: un point délimite une phrase autonome ${ }^{22}$. L'emploi de la ponctuation n'est donc pas entièrement aléatoire, même si les cas de I-b et I-c sont devenus plus fréquents que ce qu'exigeraient les règles du «bon usage»: peut-être s'agit-il plus d'omettre la ponctuation que d'en ajouter si l'on compare à la norme traditionnelle de l'écrit.

\subsubsection{Catégorie V: réduction de la proposition à une forme verbale non finie}

Pour la catégorie V (65 occurrences, soit 7,9\%), la distinction entre les deux types V-a et $\mathrm{V}$-b repose sur la présence ou non d'un joncteur, mais surtout sur le fait que V-b représente presque toujours une contrainte grammaticale (voir le tableau 2), alors que dans $\mathrm{V}-\mathrm{a}$, la forme non finie du verbe relève généralement du choix délibéré de l'auteur, contribuant ainsi à la recherche d'un style soigné.

[11] [V-a] Etant actuellement, dans une situation délicate je me permets de faire appel à vous $[\ldots]$

[12] [V-b] Je voudrais rajouter un troisième volet à ce protocole pour clarifier la situation: [...] $(1 ; 11)$

La seule occurrence dans la catégorie V-b qui ne représente pas une contrainte grammaticale est la suivante:

[13] 5 mois après environ, je reçois un recommandé de l'acheteur [...]; me demandant donc réparation.

$(2 ; 21)$

Le joncteur donc n'est pas une préposition.

\subsubsection{Catégories VI et VII: nominalisation d'un constituant avec ou sans joncteur}

Ces catégories, où une proposition (précédée ou non d'un joncteur) est remplacée par un SN, représentent le pôle intégratif de l'axe. De nombreux joncteurs introduisent un tel SN (sans, après, et, malgré, pour [dans le sens de «à cause de» et «en but de»],

21. Trois occurrences sur les neuf de cette catégorie sont des propositions séparées par des parenthèses: «(vu qu'elle est a son nom)» $(1 ; 6)$.

22. Remarquons en passant que dans les écrits de nos étudiants en FLE (français langue étrangère) des universités d'Åbo Akademi et de Tampere, les propositions subordonnées sont fréquemment délimitées par des points. 
avant, entre autres). Voici deux exemples (le SN qui suit le joncteur pourrait aussi bien être une proposition à verbe fini) :

[14] Ma femme a décidé d'une séparation définitive entre nous après une rencontre avec un autre homme.

$(1 ; 11)$

[15] Pour cette conception, je lui avais apporté des diamants.

$(2 ; 5)$

Il est impossible d'estimer le nombre exact de ces cas, mais l'emploi de ces constructions semble parfaitement naturel dans ces lettres. Il ne s'écarte nullement de l'usage «stéréotypé» du français et semble être conforme aux habitudes de l'écrit conventionnel. La présence de ces constructions contribue à la densité des lettres.

\subsubsection{Nature des joncteurs}

Ce qui est particulièrement notable dans le corpus, c'est le choix des conjonctions ou connecteurs. Il ressort de l'étude que les auteurs des lettres ont tendance à employer des connecteurs d'un registre relativement recherché, et plusieurs connecteurs du registre neutre sont quasi absents: même si (concession) n'apparait pas dans le corpus, parce que (cause) une seule fois et quand (temps) trois fois. Pour exprimer la cause, les auteurs des lettres utilisent le plus fréquemment car (31 occurrences), considéré comme "plus abstrait et moins courant que parce que» (Rey-Debove et Rey, 2011: sous «Car») ${ }^{23}$, avec (en) sachant que, étant donné que et puisque. Le participe présent est également choisi de manière récurrente (env. 10 occurrences) pour exprimer la cause (voir l'exemple de la catégorie $\mathrm{V}$-a dans le tableau 2). Par ailleurs car, au lieu d'introduire une cause, introduit parfois un commentaire énonciatif justifiant les propos de l'auteur et explicitant le fil de sa pensée ${ }^{24}$ :

[16] aujourdhui ma question est comment expulser son frere car il se vante bien quil est mineur et quil nepeut etre expulser?

$(1 ; 12)$

Pour la concession sont utilisés alors que et malgré. D’autres connecteurs qui n'apparaissent guère que dans la langue soutenue sont or (une fois écrit «hors», voir l'exemple [18]), à savoir que, afin que/de et seulement (pour exprimer la restriction). Voici des exemples:

[17] Etant donné que je reste au domicile conjugal, J'ai peur qu'elle puisse me réclamer une contribution financière égale à la moitié de la valeur locative.

$(1 ; 11)$

23. Pour une comparaison de l'emploi de car et de parce que à l'oral et à l'écrit, voir Simon et Degand (2007).

24. Voir Zufferey et al. (2018) pour une discussion des différents emplois de car et de parce que. 
[18] Mon père devait hérité de cette maison au décès de notre grand-mère hors il est partit avant elle et le testament à du être modifié.

$(1 ; 20)$

Ce caractère formel de l'écrit est sans doute aussi lié à ce discours spécialisé, le discours juridique, et à la représentation qu'en ont les auteurs des lettres.

\subsubsection{Positionnement des joncteurs}

La très grande majorité des joncteurs se trouve en début de proposition et indique donc clairement le transfert d'une proposition à l'autre. Il y a de rares occurrences d'éléments non initiaux qui indiquent les relations sémantiques entre les propositions:

[19] Le frère de mon père est légataire universel, il obtient donc une part supplémentaire $(1 ; 20)$

[20] Après avoir refusé la bague dans un premier temps (un des anneaux $\mathrm{n}$ avait pas la bonne couleur), j ai enfin récupéré ma bague mais elle était encore trop grande $(1 \mathrm{mois}$ après accouchement).

$$
(2 ; 5)
$$

Toutefois, généralement, les joncteurs qui relient des propositions entre elles se placent au point exact de liaison, et ne tissent pas une texture aussi solide que le feraient des joncteurs s'insérant à l'intérieur des propositions reliées (voir aussi infra). Le tissage reste moins serré, plus lâche, plus superficiel.

\section{Densité / desserrement}

Comme nous l'avons constaté, le tableau 2 basé sur la théorie de jonction de Raible rend compte de l'axe de l'agrégation: les prédications sont-elles rendues par des verbes finis? Quel est le degré d'intégration entre elles? Les trois premières catégories du tableau, qui présentent chacune une organisation parataxique des propositions, recouvrent dans leur ensemble $85,8 \%$ de toutes les jonctions. Le taux des catégories IV et $\mathrm{V}$, regroupant les jonctions avec une organisation hypotaxique, avec éventuellement une forme verbale réduite (participe ou infinitif), ne s'élève au total qu'à $14,2 \%$. De ce point de vue, les textes du corpus se rapprochent du pôle de l'agrégation.

Lors de la discussion ci-dessus (dans la section 4), il est devenu clair que l'axe de densité ou de desserrement rejoint l'échelle de Raible mais ne la reproduit pas, car tout ce qui contribue à la cohésion entre propositions sert à tisser la texture, qui devient plus ou moins dense. En particulier, pour parler de la densité, la métaphore du tissage est appropriée. Il faut avoir des liens de différents types, reliant les différents niveaux entre eux. Un joncteur qui présente une relation anaphorique en plus de la relation jonctive permet de créer un texte plus dense. De même, un joncteur situé en milieu de proposition tisse un texte plus dense que lorsqu'il est 
situé à la limite de deux propositions. La prédominance de la catégorie I par rapport aux autres catégories révèle pour sa part un certain desserrement dans les liens de tissage de ces lettres.

Nous proposons donc la définition suivante : la densité ici recherchée est constituée à la fois de l'intégration selon Raible et de la cohésion permettant de relier les propositions entre elles.

\section{Pour conclure}

Cette étude s'est donné comme but d'analyser le genre des lettres écrites par des internautes à l'attention de professionnels du droit, avec un intérêt particulier porté sur les jonctions entre propositions. Comme nous l'avons vu, les textes du corpus ont de nombreuses caractéristiques de textes écrits conventionnels, notamment une cohérence claire qui suit un certain modèle. Plusieurs traits les distinguent toutefois du genre de lettre plus traditionnel auquel nous pensions qu'ils tendaient.

Les lettres étudiées révèlent la présence de deux traditions discursives: la tradition conventionnelle appartenant au français écrit du «bon usage» et une tradition plus novatrice, se développant depuis la généralisation des nouveaux supports électroniques d'écriture. Le genre de la «lettre officielle de demande d'aide à un juriste» est une combinaison opérative des deux.

De même, en ce qui concerne l'axe de densité/desserrement que nous avons étudié via les jonctions entre propositions, le genre discursif en question est à la charnière de tendances presque opposées. La densité du français écrit conventionnel, répondant à des choix esthétiques traditionnels, est confrontée à de multiples moyens linguistiques plus lâches.

Cette étude aura permis de connaitre un peu mieux la structure propositionnelle dans des textes "post-numériques» et de poser les premiers jalons qui pourront servir de point de comparaison pour des études ultérieures. Les développements actuels et futurs de l'esthétique de l'écriture, en partie dévoilés ici, restent à être étudiés dans d'autres travaux.

\section{Références bibliographiques}

Adam, J.-M. 2015. La linguistique textuelle. Cursus. Paris: A. Colin [3éd.].

Barats, C. (éd.) 2013. Manuel d'analyse du web en sciences bumaines et sociales. Paris: A. Colin. Berrendonner, A. 1990. Pour une macro-syntaxe. Travaux de linguistique 21: 25-36.

Biber, D. 1995. Dimensions of Register Variation: A Cross-Linguistic Comparison. Cambridge New York: Cambridge University Press.

Biber, D. 2006. University Language: A Corpus-Based Study of Spoken and Written Registers. Studies in corpus linguistics 23. Amsterdam - Philadelphie: J. Benjamins.

Blanche-Benveniste, C. (éd.) 1990. Le français parlé: études grammaticales. Paris: CNRS. 
Blanche-Benveniste, C. 2010. Approches de la langue parlée. Paris: Ophrys [nouv. éd.].

Brunot, F. 1966. Histoire de la langue française des origines à nos jours. Paris: A. Colin. T. III : La formation de la langue classique 1600-1660.

Buysse, M. 2016a. L'acquisition de la liaison de propositions en langue seconde: traitement et apport de la "Role and Reference Grammar». Thèse de doctorat. Université de HelsinkiVrije Universiteit Brussel.

Buysse, M. 2016b. Dépendance sans intégration: la cosubordination comme mode de jonction propositionnelle et sa pertinence en acquisition du français L2. In F. Neveu, G. Bergounioux, M.-H. Côté, J.-M. Fournier, L. Hriba et S. Prévost (éd.), SHS Web of Conferences. Actes du $5^{e}$ congrès mondial de Linguistique française - CMLF 2016 (Tours, 4-8 juillet 2016). Les Ulis: EDP Sciences. Vol. 27: 1-16. En ligne à l'adresse suivante: https://www.shs-conferences.org/articles/shsconf/pdf/2016/05/shsconf_ cmlf2016_10003.pdf.

Charaudeau, P. et Maingueneau, D. (éd.) 2002. Dictionnaire d'analyse du discours. Paris : Seuil.

Ferguson, C. A. 1972. Diglossia. In P. P. Giglioli (éd.), Language and Social Context: Selected Readings. Harmondsworth : Penguin: 232-251. [Parution originale: FERGuson, C. A. 1959. Diglossia. Word 15 (2): 325-340.]

GAUTIER, A. 2014. Phrase et syntaxe: sur quelques aspects de l'intégration. Langue française 182: 27-41.

Hakulinen, A. 2003. Vielä nykysuomesta ja sen huollosta. Ovatko puhuttu ja kirjoitettu kieli erkaantuneet toisistaan? Kielikello 1. En ligne à l'adresse suivante: http://www. kielikello.fi/index.php?mid=2\&pid=13\&aid=1401.

Halliday, M. A. K. et Hasan, R. 1976. Cohesion in English. Londres - New York: Longman.

Herring, S. C., Stein, D. et Virtanen, T. (éd.) 2013. Pragmatics of Computer-Mediated Communication. Handbooks of pragmatics 9. Berlin - Boston : De Gruyter.

Kabatek, J., Obrist, P. et Vincis, V. 2010. Clause-Linkage Techniques as a Symptom of Discourse Traditions: Methodological Issues and Evidence from Romance Languages. In H. Dorgeloh et A. Wanner (éd.), Syntactic Variation and Genre. Topics in English linguistics 70. Berlin - New York: De Gruyter: 247-275.

КосH, P. 1995. Subordination, intégration syntaxique et «oralité». In H. L. ANDERSEN et G. Sкүтte (éd.), La subordination dans les langues romanes. Études romanes 34. Copenhague: Munksgaard International Publishers: 13-42.

Koch, P. et Oesterreicher, W. 1985. Sprache der Nähe - Sprache der Distanz: Mündlichkeit und Schriftlichkeit im Spannungsfeld von Sprachtheorie und Sprachgeschichte. Romanistisches Jabrbucb 36 : 15-43.

Koch, P. et Oesterreicher, W. 1994. Schriftlichkeit und Sprache. In H. GüNTHER et O. LudwIG (éd.), Schrift und Schriftlicbkeit: ein interdisziplinäres Handbuch internationaler Forschung - Writing and Its Use: An Interdisciplinary Handbook of International Research. Berlin - New York: De Gruyter. Vol. I: 587-604.

Koch, P. et Oesterreicher, W. 2011 [1990]. Gesprochene Sprache in der Romania: Französisch, Italienisch, Spanisch. Romanistische Arbeitshefte 31. Berlin - New York: De Gruyter [2 éd. remaniée]. 
Lefeuvre, F. 1999. La phrase averbale en français. Paris: L'Harmattan.

Lehmann, C. 2011 [1988]. Towards a Typology of Clause Linkage. In J. Haiman et S. A. Thompson (éd.), Clause Combining in Grammar and Discourse. Amsterdam Philadelphie: J. Benjamins: 181-225.

Lodge, R. A. 1997 [1993]. Le français: bistoire d'un dialecte devenu langue. C. VeKEN (trad.). Paris: Fayard.

Luzzati, F. et Luzzati, D. 1987. Oral et familier: le style oralisé. L'information grammaticale $34: 15-21$.

Maingueneau, D. 2014. Aux limites de la généricité. In M. Monte et G. Philippe (éd.), Genres et textes - déterminations, évolutions, confrontations: études offertes à Jean-Michel Adam. Textes et langue. Lyon: Presses universitaires de Lyon : 77-88.

Marcoccia, M. 2016. Analyser la communication numérique écrite. Malakoff: A. Colin.

Matthiessen, C. et Thompson, S. A. 2011 [1988]. The Structure of Discourse and "Subordination". In J. Haiman et S. A. Thompson (éd.), Clause Combining in Grammar and Discourse. Amsterdam - Philadelphie: J. Benjamins: 275-329.

Paveau, M.-A. 2017. L'analyse du discours numérique: dictionnaire des formes et des pratiques. Paris: Hermann.

Paveau, M.-A. et Rosier, L. 2008. La langue française: passions et polémiques. Paris: Vuibert.

PhilipPe, G. et Piat, J. (éd.) 2009. La langue littéraire: une bistoire de la prose en France de Gustave Flaubert à Claude Simon. Paris: Fayard.

Raible, W. 1992. Junktion: eine Dimension des Sprache und ibre Realisierungsformen zwischen Aggregation und Integration. Sitzungsberichte der Heidelberger Akademie der Wissenschaften, Bericht 2. Heidelberg: C. Winter.

Raible, W. 2001. Linking Clauses. In M. Haspelmath, E. KöNig, W. Oesterreicher et W. Raible (éd.), Language Typology and Language Universals: An International Handbook. Berlin - New York: De Gruyter. Vol. I: 590-617.

Rey-Debove, J. et Rey, A. (éd.) 2011. Le petit Robert. Paris: Dictionnaires Le Robert.

Riegel, M., Pellat, J.-C. et Rioul, R. 2009 [1994]. Grammaire méthodique du français. Paris: PUF [ $4^{\mathrm{e}}$ éd.].

Simon, A. C. et Degand, L. 2007. Connecteurs de causalité, implication du locuteur et profils prosodiques: le cas de car et de parce que. Journal of French Language Studies 17 (3) : 323-341.

Walsh, O. 2016. Linguistic Purism: Language Attitudes in France and Quebec. Impact Studies in Language and Society 41. Amsterdam - Philadelphie: J. Benjamins.

Welcomme, A. 2012. La jonction interpropositionnelle à l'oral et à l'écrit en français langue étrangère. Thèse de doctorat. Vrije Universiteit Brussel.

Zufferey, S., MaK, W., Verbrugge, S. et SAnders, T. 2018. Usage and Processing of the French Causal Connectives Car and Parce que. Journal of French Language Studies 28 (1): 85-112. 